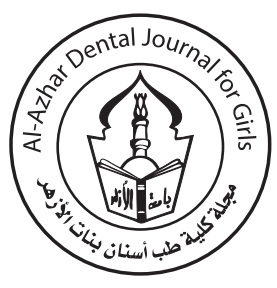

\title{
Clinical Evaluation of Nano-Hydroxyapatite as Dentin Desensitizer
}

\author{
Shimaa M. Ameen ${ }^{(1)}$, Maha A. Niazy ${ }^{(2)}$, Mohamed A. El-yassaky ${ }^{(2)}$, Wael E. Jamil ${ }^{(3)}$, Mai S. Attia ${ }^{(4)}$
}

Codex : 10/1801

azhardentj@azhar.edu.eg

http://adjg.journals.ekb.eg

\section{KEYWORDS}

Nano-Hydroxyapatite

Dentin Desensitizer

\begin{abstract}
Objectives: This clinical study was conducted to evaluate the efficiency of nanohydroxyapatite (n-HAp) in reducing dentin hypersensitivity individually or combined with fluoride. Materials and methods: Forty hypersensitive teeth from 10 patients with hypersensitive symptoms were treated using hydroxyapatite nanoparticles (nHAp) gel at different concentrations/ sodium fluoride $(\mathrm{NaF})$ containing according to four treatment groups: Group 1; $15 \%$ nHAp $+1 \%$ NaF, Group 2; $15 \%$ nHAp, Group 3; $25 \% \mathrm{nHAp}+1 \% \mathrm{NaF}$, Group 4; $25 \%$ nHAp. The initial sensitivity levels were recorded using a verbal rating scale (VRS) and visual analogue scale (VAS) at the baseline visit. The responses were evaluated at 0,1 day, 1, 2 and 4 weeks respectively for all groups. Results: There was a high statistical significant differences between pain scores before and after treatment for all treated groups. There was statistically significantly high difference between $25 \% \mathrm{nHAp}$ and the other groups, as they need 4 applications for complete relief while $25 \%$ nHAp needs only 3 applications. Conclusion: Nanohydroxyapatite with $25 \%$ concentration was the most effective in alleviating the hypersensitivity symptoms.
\end{abstract}

\section{INTRODUCTION}

Life expectancy is increasing and patients are retaining their natural teeth for a longer time due to effective treatment strategies for caries and periodontal disease. Consequently, there is a higher risk of cervical dentin hypersensitivity as a result of physiological gingival recession with aging ${ }^{(1)}$. Dentin hypersensitivity is defined as pain arising from exposed cervical dentin caused by chemical (erosive foods and drinks), thermal (hot and cold), mechanical (brushing), evaporative or osmotic

1. Lecturer of Operative Dentistry, Faculty of Dental Medicine for Girls, AL-Azhar University

2. Professor of Operative Dentistry, Faculty of Dental Medicine for Girls, AL-Azhar University

3. Associate professor of Operative Dentistry, Faculty of Dental Medicine for Girls, AL-Azhar University

4. Associate professor of Oral Medicine, Periodontology, Diagnosis and Oral Radiology, Faculty of Dental Medicine for Girls, AL-Azhar University 
stimuli applied to opened dentinal tubules. This is a common problem found in many adult populations, with a prevalence ranging from 8 to $57 \%$. Dentinal tubules play an important role in transferring stimuli and irritants to the pulp. The hydrodynamic theory of dentin sensitivity postulates a flow of fluid through the tubule as the transducing mechanism for hydrodynamic stimuli ${ }^{(2,3)}$.

It has been demonstrated that hypersensitive dentin has a larger number of wide, open dentinal tubules on the surface than does non-sensitive dentin. A rational approach to the control of pain arising from exposed dentin would thus be to block or reduce the diameter of the tubules. Occluding dentinal tubular agents can create a barrier by precipitating proteins and calcium/phosphate ions on the surface or within the tubule orifices. The mechanism of action of the various chemical desensitizing agents is still not well understood, as these agents might have different behavior when applied to in vitro and in vivo conditions ${ }^{(1,3)}$. A number of treatment options are currently available for the treatment of hypersensitivity. These range from the commercially available, over-the-counter dentifrices containing potassium nitrate, strontium chloride, or fluorides, to the in-office treatment procedures, which include iontophoresis and application of resins and adhesives. These agents may bring about therapeutic effects either by partial or complete obturation of dentinal tubules (tubule occluding agents), by anti-inflammatory activity, by protein precipitation, or by sealing the tubules ${ }^{(4)}$.

Recently, research into the use of the mineral components of the inorganic portion of the tooth structure (i.e., calcium and phosphate) has evoked considerable interest and has inspired the preparation of hydroxyapatite (HAp) for occlusion of dentinal tubules. HAp is the main constituent of teeth and bones and hence very desirable as a natural occluding material ${ }^{(5,6)}$. Therefore this clinical study was conducted to evaluate the desensitizing effect of different concentrations and applications of nanohydroxyapatite (nHAp) individually or combined with fluoride.

\section{MATERIALS AND METHODS}

\section{Materials:}

\section{A. Materials used as dentin desensitizers:}

Four different nano- Hydroxyapatite (nHAp) gel preparation were used in this study:

1. $15 \%$ Nano-Hydroxyapatite (nHAp) $+1 \%$ sodium fluoride $(\mathrm{NaF})$.

2. $15 \%$ Nano-Hydroxyapatite (nHAp).

3. $25 \%$ Nano-Hydroxyapatite (nHAp) $+1 \%$ sodium fluoride $(\mathrm{NaF})$.

4. $25 \%$ Nano-Hydroxyapatite (nHAp).

\section{B. Nano hydroxyapatite (nHAp) powder preparation:}

Hydroxyapatite nanoparticles had been prepared in Nanotech Egypt by wet chemical method (7). Hydroxyapatite nanoparticles were formed through the wet chemical reaction of calcium nitrate, with ammonium phosphate $\left(\left(\mathrm{NH}_{4}\right) \mathrm{HPO}_{4}\right)$ the grain size was controlled by changing the time and the temperature of HAp precipitation. The $\mathrm{pH}$ values were adjusted between 10 and 12 and the reaction was performed at room temperature $\left(20^{\circ} \mathrm{C}\right)$.

\section{Characterization:}

- Size \& Shape:

Powder was examined using TEM (JEOL JEM-2100) high resolution transmission electron microscope at an accelerating voltage of $200 \mathrm{kV}$. The TEM micrographs show that, the prepared HA NPs has Rod-like shape and the size ranged between $100 \pm 20 \mathrm{~nm}$ (L), $20 \pm 10 \mathrm{~nm}$ (D) (Fig.1).

\section{Optical Properties:}

FT-IR spectrum were performed on Bruker FTIR spectrometer with range of $\left(4000-400 \mathrm{~cm}^{-1}\right)$, and resolution (less than $0.1 \mathrm{~cm}^{-1}$ ). 


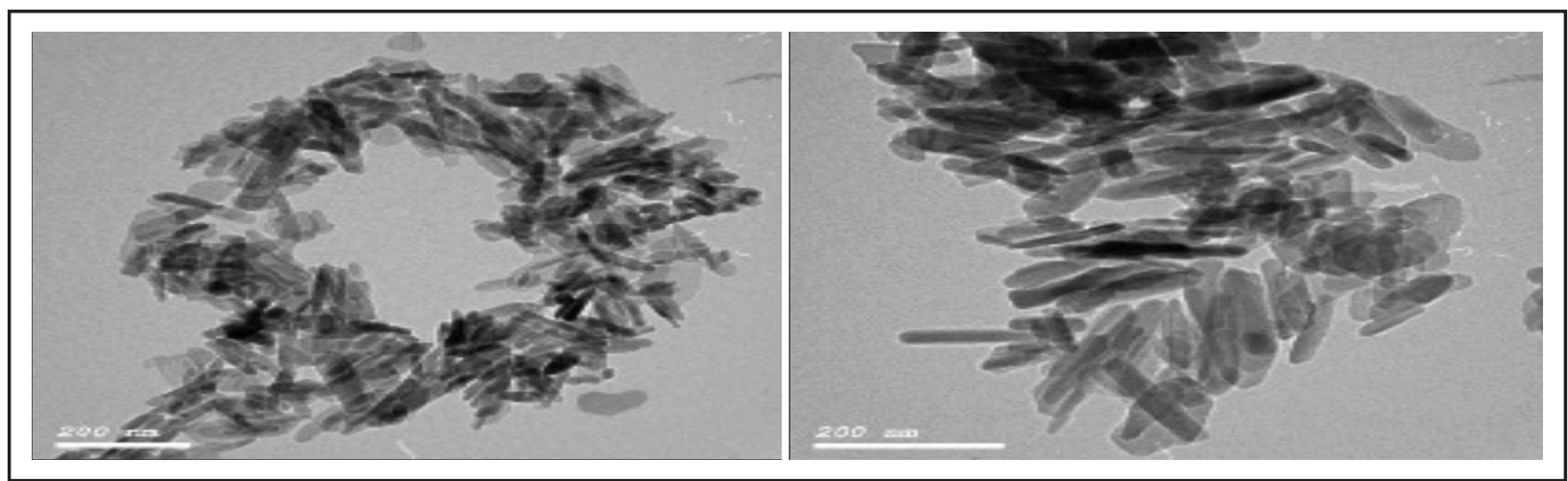

Fig. (1) TEM micrograph of nHAp particles.

\section{Nano hydroxyapatite (nHAp) gel preparation:}

The prepared nano hydroxyapatite powder was formed into gel in (Nanotech Egypt) as follow: The nHAp was dispersed in the initiator solution $(0.4 \%$ IRGACURE 2959 dissolved in deionized water) and dissolved by vortexing for $10 \mathrm{~min}$. and sonicated for $20 \mathrm{~min}$. more to obtain a uniform distribution of nanoparticles. Then, the polyethylene glycol diacrylate (PEG diacrylate) (Sigma-Aldrich) was added to the nHAp solution and allowed to be mixed for $20 \mathrm{~min}$. Finally, the mixture was centrifuged for $1 \mathrm{~min}$. at $200 \mathrm{rpm}$ to remove any air bubbles. There was no phase separation after centrifugation. The composite solution was injected into a mold and then photo-cross-linked for $10 \mathrm{~min}$. using a high-intensity UV lamp (B-100AP, Ultra-Violet Products, Upland, CA, wavelength used: $365 \mathrm{~nm}$ ). The distance between the sample and the UV lamp was kept at $15 \mathrm{~mm}{ }^{(8)}$.The composition of the nanohydroxyapatite gels (by weight) is listed in table (1).

\section{Methods:}

A double blind clinical trial with split mouth design was conducted according to the American Dental Association (ADA) guidelines for the acceptance of products for the treatment of dentinal hypersensitivity.

\section{A. Case selection:}

Forty (40) hypersensitive teeth from ten (10) patients ( 3 males and 7 females) were enrolled for this study. Patients' age ranged from $(20-45)$ years old. Each of the 10 patients had their hypersensitive teeth in the anterior and premolar areas. A special patient chart was designed to have a systematic and methodic recording of all the observations and information, which included a detailed case history, clinical examination, relevant information related to the hypersensitivity and precipitating causes, Verbal Rating Scale (VRS) scores and Visual Analogue Scale (VAS) scores to facilitate recall visits.

Table (1): Composition of nano-hydroxyapatite gel.

\begin{tabular}{|c|c|c|c|c|}
\hline Samples & nHApwt \% & NaFwt \% & PEG wt $\%$ & Water wt \% \\
\hline 0\% nHAp & 0 & 0 & 20 & 80 \\
\hline $15 \%$ nHAp & 15 & 0 & 20 & 65 \\
\hline $15 \%$ nHAp+1\% NaF & 15 & 1 & 20 & 64 \\
\hline $25 \%$ nHAp & 25 & 0 & 20 & 55 \\
\hline $25 \%$ nHAp $+1 \%$ NaF & 25 & 1 & 20 & 54 \\
\hline
\end{tabular}


Patient selections were according to inclusion/ exclusion criteria $^{(9)}$. Also charting was made for each patient along with a written consent of the patient for willingness to participate in the study.

\section{B.Patients preparations:}

The patients initially completed a plaque control program, including oral hygiene instruction, scaling and root planning. The patients were instructed to perform non-traumatic brushing technique twice a day for 2 min and not to use any desensitizing agent or dentifrice. Only those patients maintaining optimum oral hygiene as well as showing compliance towards the correct brushing technique were included in the study ${ }^{(10)}$.

\section{C.Subjective assessment of dentin hypersensitivity:}

The subjective assessment of dentin hypersensitivity was done before application of the agent (baseline) then immediate after treatment, 1day, 1 week, 2 weeks and 4 weeks respectively. The patients were educated to rate their perception to tactile, air and cold stimuli by using the VRS ${ }^{(11)}$.

\section{a. Verbal Rating Scale (VRS)}

The degree of hypersensitivity was determined according to the Verbal Rating Scale - VRS. The patient uses a numerical code from 0 to 4 to rate perceived sensation, in which:

- Score 0: Was recorded for those patients with No discomfort.

- Score 1: Was recorded for patients complaining from mild discomfort.

- $\quad$ Score 2: Was recorded for patients complaining from moderate discomfort.

- Score 3: Was recorded for patients complaining from severe pain only during application of stimulus.

- Score 4: Was recorded for patients complaining from severe pain persisting after removal of stimulus.
The following stimuli were applied for clinical evaluation:

1. Probing stimulus: A sharp dental explorer was passed over the affected area, perpendicular to the long axis of the tooth without any pressure. All stimuli were performed after all subjected teeth had been cleaned with a cotton pellet soaked in warm distilled water, and dried.

2. Air blast: This method was performed two minutes after the tactile scoring procedure. A blast of air from a dental syringe attached to dental unit was directed onto the affected area of the tooth in a distance of $1 \mathrm{~cm}$ from the tooth surface to avoid desiccating the dentin surface. This stimulus was delivered until a reaction was observed, or up to a maximum duration of 5 seconds ${ }^{(9)}$. All stimuli were performed by the same operator, in the same dental chair with the same equipment while the pain was assessed with the VAS (Visual Analogue Scale) by another clinician. The patients' reactions to the stimuli were recorded before and immediately after treatment, and then at one-day, 1-week, 2weeks and 4-weeks.

3. Thermal stimulus (cold stimulus): Drops of melting ice held in a plastic syringe were made to flow over the sensitive tooth surface for five seconds.

\section{b. Visual Analogue Scale (VAS):}

The patient scores pain intensity on a 10$\mathrm{cm}$ straight line scale traced on a piece of paper. Specifically, Patients recorded their overall assessment of tooth sensitivity that resulted from their normal daily activities. This was done using a VAS. The scale consisted of a 100-mm straight line scale traced on colored bar. Participants were instructed to mark the far left hand end of the line if the pain was "the worst you could imagine," or, if pain was less than that, to "place a mark anywhere along the line that represented what you felt." The score was determined by measuring from the left hand edge of the line to the point where the participant's mark intersected the line. Scores were measured 
and recorded to the nearest millimeter. Completed logs were collected at each evaluation. Specifically, $0=$ no pain and $10=$ extreme, unbearable pain .

\section{The clinical procedures:}

Hypersensitive teeth in each patient were randomly divided into four groups as stated previously. Total of 40 teeth in split mouth design were selected and divided into 4 groups treated with the four different nHAp gel preparations either with or without sodium fluoride.

The area to be treated was isolated with cotton rolls and was dried using air spray. The agents were injected with sterile needle tip at the cervical region of the tooth for 1minute. Patients were instructed not to rinse or to take any food stuff for half an hour. This treatment was repeated at 1 day, 1 week, 2 weeks and 4 weeks. The patients were demonstrated the proper brushing technique and were advised to use a soft toothbrush. Diet counseling was also done in order to avoid intake of excessive dietary acids during the study period. ${ }^{(12)}$.

\section{Statistical Analysis}

IBM SPSS statistics (V. 22.0, IBM Corp., USA, 2013) was used for data analysis. Data were expressed as Mean $\pm \mathrm{SD}$ for quantitative parametric measures. The following tests were done:

1. The degree of change due to follow-up study (delta change or $\mathrm{dC}$ ) reflect the actual diff. changed through the follow-up study and can be calculated for each patient, from which, the mean delta change can compared with other group or correlate with other variables. It is defined as follow: Delta change $(\mathrm{dC})=($ Post-Pre $) /$ Pre .

2. Comparison between more than 2 patient groups for parametric data using Analysis of Variance (ANOVA). The multiple comparisons (Posthoc test or least significant difference, LSD) were also followed to investigate the possible statistical significance between each 2 groups. The probability of error at 0.05 was considered sig., while at 0.01 and 0.001 are highly significant.

\section{RESULTS}

The degree of hypersensitivity was determined according to Verbal Rating Scale (VRS) and Visual Analogue Scale (VAS). Forty hypersensitive teeth from ten (10) patients (7females and 3 males) completed the 4- weeks follow up.

Table (2): Statistical analysis using Analysis of variance for comparison between VRS \&VAS in the four groups.

\begin{tabular}{|c|c|c|c|c|c|c|c|c|c|}
\hline \multicolumn{10}{|c|}{ Probing stimulus } \\
\hline \multirow{2}{*}{ Aroup } & \multicolumn{2}{|c|}{$\begin{array}{c}15 \% \text { nHAP+ } \\
1 \% \mathrm{NaF}\end{array}$} & \multicolumn{2}{|c|}{$\begin{array}{c}15 \% \\
\text { NHAP } \\
\end{array}$} & \multicolumn{2}{|c|}{$\begin{array}{c}25 \% \text { nHAP+ } \\
1 \% \mathrm{NaF}\end{array}$} & \multicolumn{2}{|c|}{$\begin{array}{l}25 \% \\
\text { nHAP }\end{array}$} & \multirow{2}{*}{ P- value } \\
\hline & Mean & SD & Mean & SD & Mean & $\mathrm{SD}$ & Mean & $\mathrm{SD}$ & \\
\hline Before treatment & 2.2 & 0.52 & 2.4 & 1.03 & 2.3 & 0.52 & 2.8 & 1.03 & 0.502 \\
\hline Immediate after treatment & 0.4 & 0.52 & 0.4 & 0.52 & 0.8 & 0.52 & 0.4 & 0.52 & 0.472 \\
\hline After 1 day treatment & 0 & 0 & 0 & 0 & 0 & 0 & 0 & 0 & \\
\hline After 1week & 0 & 0 & 0 & 0 & 0 & 0 & 0 & 0 & \\
\hline After 2 weeks & 0 & 0 & 0 & 0 & 0 & 0 & 0 & 0 & \\
\hline After 4 weeks & 0 & 0 & 0 & 0 & 0 & 0 & 0 & 0 & \\
\hline
\end{tabular}




\begin{tabular}{|c|c|c|c|c|c|c|c|c|c|}
\hline & & & & iulus & & & & & \\
\hline Application & $15 \% \mathrm{nH}$ & $1 \% \mathrm{NaF}$ & $15 \%$ & AAP & $25 \% \mathrm{nH}$ & $1 \% \mathrm{NaF}$ & 259 & IAP & P-value \\
\hline Group & Mean & SD & Mean & SD & Mean & SD & Mean & SD & $0.001 * *$ \\
\hline Before treatment & $2.6_{b}$ & 0.52 & 3 a & 0 & $2.6_{b}$ & 0.52 & $3 a$ & 0 & $0.018 *$ \\
\hline Immediate after treatment & $1.8 \mathrm{a}$ & 0.7 & $1.8_{\mathrm{a}}$ & 0 & $1.6_{\mathrm{a}}$ & 0.84 & $1_{\mathrm{b}}$ & 0.42 & $0.004 *$ \\
\hline After 1 day treatment & $1_{\mathrm{a}}$ & 0.94 & $0.2_{\mathrm{b}}$ & 0.42 & 0.8 a & 0.79 & $0_{\mathrm{b}}$ & 0 & $0.036^{*}$ \\
\hline After 1 week & $0.6_{\mathrm{a}}$ & 0.52 & $0.4_{\text {a }}$ & 0.52 & $0.4_{\mathrm{a}}$ & 0.52 & $0_{\mathrm{b}}$ & 0 & \\
\hline After 2 weeks & 0 & 0 & 0 & 0 & 0 & 0 & 0 & 0 & \\
\hline After 4 weeks & 0 & 0 & 0 & 0 & 0 & 0 & 0 & 0 & \\
\hline & & & $\mathrm{Co}$ & timulu & & & & & \\
\hline Before treatment & $2.6 \mathrm{~b}$ & 0.52 & $3 a$ & 0 & $2.4 \mathrm{~b}$ & 0.52 & $3 a$ & 0 & $0.001 * *$ \\
\hline Immediate after treatment & 1.8 & 0.79 & 1.6 & 0.52 & 1.6 & 0.84 & 2 & 0 & 0.443 \\
\hline After 1 day treatment & 1 & 0.94 & 0.6 & 0.52 & 0.8 & 0.79 & 0.6 & 0.52 & 0.548 \\
\hline After 1 week & $0.6 \mathrm{~b}$ & 0.52 & $0.4 \mathrm{ab}$ & 0.52 & $0.6 \mathrm{~b}$ & 0.52 & $0 \mathrm{a}$ & 0 & $0.015^{*}$ \\
\hline After 2 weeks & 0 & 0 & 0 & 0 & 0 & 0 & 0 & 0 & \\
\hline After 4 weeks & 0 & 0 & 0 & 0 & 0 & 0 & 0 & 0 & \\
\hline & & & & AS & & & & & \\
\hline Before treatment & $6.4 \mathrm{~b}$ & 1.26 & $6.6 \mathrm{~b}$ & 0.52 & $6.2 \mathrm{~b}$ & 1.23 & $7.6 \mathrm{a}$ & 0.52 & $0.011 *$ \\
\hline $\begin{array}{c}\text { Immediately after } \\
\text { treatment }\end{array}$ & 4.4 & 1.27 & 3.6 & 0.52 & 4.2 & 1.23 & 4.2 & 0.79 & 0.324 \\
\hline After 1 day treatment & 2 & 1.76 & 1.6 & 1.43 & 2 & 1.76 & 1.2 & 1.03 & 0.601 \\
\hline After 1 week & $1.2 \mathrm{a}$ & 1.03 & $0.8 \mathrm{ab}$ & 1.03 & $1.2 \mathrm{a}$ & 1.03 & $0 \mathrm{~b}$ & 0 & $0.015^{*}$ \\
\hline After 2 weeks & 0 & 0 & 0 & 0 & 0 & 0 & 0 & 0 & \\
\hline After 4 weeks & 0 & 0 & 0 & 0 & 0 & 0 & 0 & 0 & \\
\hline
\end{tabular}

*: significant at $P \leq 0.05$, **: High significant at $P \leq 0.01$, a and $b$ : The same letter indicates no significant difference in the same row at $\alpha=0.05$ by Tukey's multiple comparison test.

\section{DISCUSSION}

Dentin hypersensitivity is a very common painful problem which is difficult to solve, despite the fact that a large variety of treatments exist. The occurrence of hypersensitivity is due to abrasion, abfraction, particularly important as an adverse event of periodontal therapy. It has been demonstrated that root exposure due to loss of attachment and shrinkage of periodontal tissues leads to exposure of the cemento-enamel junction and tooth hypersensitivity ${ }^{(13,14)}$. Taking these facts into consideration, there is a need to develop treatment approaches which permit the relief of the symptoms of dentine hypersensitivity. 
Hydroxyapatite is a natural mineral constituent of teeth and bones, and hence the use of this material as a desensitizing agent to occlude dentinal tubules was highly desirable. The motivation for synthesizing HAP by different means has been to obtain Nano sized particles for infiltration of dentinal tubules, for the alleviation of hypersensitivity. It is clear that in vivo experiment is the ideal methodology for biological investigation and in vitro studies can only address limited aspects of any natural system ${ }^{(15)}$.

Utilizing safe and effective biological materials through their physical or chemical properties to block exposed dentinal tubules, to reduce or inhibit the flow of tubular fluid, and to avoid stimwulating pulp nerve endings is thus an effective means for controlling dentin sensitivities ${ }^{(16)}$.

The ultimate test of any treatment is how well it works in the clinic. A randomized, blinded and controlled trial is the gold standard for determining the efficacy. A split mouth study design was chosen in this work which had advantages of same pain perception, oral hygiene habits, dietary habits and psychosomatic factors ${ }^{(17)}$. The subject acts as his own control which is very powerful tool statistically, and the methodology of choice ${ }^{(18)}$.

Ideally, the evaluation period in the clinical study should have been longer than four weeks; however, as the goal of the study was to determine which agents would eliminate the participant's acute complains of DH rapidly and effectively, a short-term (four-week) study was conducted. It is important, however, in that studies be carried out to determine which agents provide long-term relief from $\mathrm{DH}$. Although most hypersensitive teeth are sensitive to more than one stimulus, not all teeth respond to the same stimulus ${ }^{(19)}$.

Based on the recommendations of Ad Hoc Advisory Committee on Dentinal Hypersensitivity ${ }^{(20)}$, three different stimuli- tactile, air and cold water were used to assess hypersensitivity in the study. All suspected dental sites were investigated by using a probe tip as a tactile stimulus, which causes the inward movement of the dentinal fluid owing to the compression of the dentin. Thus, mechanoreceptors causing the painful sensation were activated ${ }^{(21)}$.

Air stimulus decreases the temperature at the dentin surface, causes a rapid outward fluid flow from opened dentin tubules, which stimulates the painful sensation ${ }^{(22,23)}$. Currently no single method of eliciting and assessing cervical dentin sensitivity may be considered ideal, and hence, a combined assessment using two different methods seemed appropriate to eliminate the deficiencies of either one method, which was why the verbal rating scale (VRS) and the Visual analogue scale (VAS) were used in the present study. The VRS offers a restrictive choice of words, which may not represent the pain experience, with significant precision for all patients ${ }^{(24)}$. On the other hand, the validity and reliability of the VAS for both experimental and clinical pain has been demonstrated by several investigators $^{(21)}$. The VAS also appears to be more sensitive in discriminating between various treatments and changes in pain intensity.

The clinical results showed significant reduction of DH immediately after treatment of all test groups at probing stimulus, air stimulus, cold stimulus and (VAS). In addition, after 4 weeks of follow up, no adverse events were reported by participants. The efficacy of the n-HAp may be explained considering the hydrodynamic theory based on the alteration of fluid flow in dentinal tubules; if the dentinal tubules are obliterated anywhere along their length, hydraulic conductance will be reduced and pain consequently diminished. Although it was observed that all the four agents did not differ significantly in their desensitizing effects at the end of 4 weeks, there were considerable statistically significant differences in the number of applications required by each of the agents in bringing about relief of hypersensitivity symptoms both at probing stimulus, 
air stimulus, cold stimulus and (VAS). Treated teeth with 25\% nHAP required fewer applications to obtain a favorable response. Most patients obtained relief within the second or third application, which was not the case with the other agents where three to four applications of the agent provided relief. This may be attributed to the difference in concentrations These clinical results were augmented by ESEM and permeability results which revealed that three applications of $25 \% \mathrm{nHAp}$ gave the best results. This further reiterates the permanent desensitizing effects of nanohydroxyapatite due to its tubule occluding and blending capacity, as suggested by other studies leading to quicker and long lasting effect ${ }^{(9)}$.

\section{CONCLUSION}

1. Nano-hydroxyapatite (nHAp) was effective in alleviating dentin hypersensitivity symptoms.

2. The clinical application of nHAp alleviated the hypersensitivity symptoms within 1 to 2 weeks (3-4 applications) and sustained up to 4 weeks.

\section{RECOMMENDATION}

1. The findings of the present study encourage the application of nHAp as dentin desensitizer. Thanks to its low cost, ease of use and its biocompatibility with no proven adverse effects, nHAp is valid with definite potential as an effective desensitizing agent providing quick relief from symptoms.

2. Although the results are encouraging, this study could be considered limited by its short term follow up so further long-term studies with a larger sample size are recommended for comparison with other more popular commercially available agents and procedures, such as lasers and ozone should be attempted.

\section{REFERENCES}

1. Rees JS and Addy MA. Cross-sectional study of dentin hypersensitivity. J Clin Periodontol. 2002; 29: 997-1003.

2. Brannstrom M, Lindén LA, Astrom A. The hydrodynamics of the dental tubule and of pulp fluid. A discussion of its significance in relation to dentinal sensitivity. Caries Res.1976; 1: 310-7.

3. Ritter AV, Dias LW, Miguez P, Caplan DJ, Swift EJ. Treating cervical dentin hypersensitivity with fluoride varnish: a randomized clinical study. J Am Dent Assoc. 2006; 137: 1013-20.

4. Gillam DG and Orchardson R. Advances in the treatment of root dentin sensitivity: Mechanisms and treatment principles. Endo Topics. 2006; 13: 13-33.

5. Ishikawa K, Suge T, Yoshiyama M, Kawasaki A, Asoaka $\mathrm{K}$, Ebisu S. Occlusion of dentinal tubules with calcium phosphate using acidic calcium phosphate solution followed by neutralization. J Dent Res. 1994; 73:1197-204.

6. Suge T, Ishikawa K, Kawasaki A, Yoshiyama M, Asaoka $\mathrm{K}$, Ebisu S. Effects of fluoride on the calcium phosphate precipitation method for dentinal tubule occlusion. J Dent Res.1995; 74:1079-85.

7. Paz A, Guadarrama D, Lopez M, Gonz.lez JE, Brizuela N, Aragon J. A comparative study of hydroxyapatite nanoparticles synthesized by different routes. Quim Nova. 2012; $35: 1724-7$.

8. Gaharwar AK, Dammu SA, Canter JM, Wu CJ, Schmidt G. highly extensible, tough, and elastomeric nanocomposite Hydrogels from Poly ethylene glycol and Hydroxyapatite Nanoparticles. Biomacromolecules. 2011; 12: 1641-50.

9. Shetty S, Kohad R, Yeltiwar R. Hydroxyapatite as an in- Office agent for tooth hypersensitivity: A clinical and scanning electron microscopic study. J Periodontol. 2010; 81:1781-89.

10. Verma P, Gupta U, Dodwad V, Kukreja B J, Arora K. Evaluation of the clinical efficacy of new desensitizing toothpaste containing nano-crystalline hydorxyapatite in dentin hypersensitivity patients: A double blind randomized controlled clinical trial. J Dent Spec. 2013; 1:47- 54.

11. Sharma S, Shetty N J, Uppor A. Evaluation of the clinical efficacy of potassium nitrate desensitizing mouth wash and a tooth paste in the treatment of dentinal hypersensitivity. $\mathrm{J}$ Clin ExpDent.2012; 4:28-33.

12. Drisko $\mathrm{CH}$. Dentin hypersensitivity- dental hygiene and periodontal considerations. Int Dent J. 2002; 52: 385-93. 
13. Bissada NF. Symptomatology and clinical features of hypersensitive teeth. Arch Oral Biol.1994;39:31-2.

14. Von Troil B, Needleman I, Sanz MA. Systematic review of the prevalence of root sensitivity following periodontal therapy. J Clin Periodontol. 2002; 29: 173- 7.

15. Sales-Peres SC, Carvahlo FN, Marsicano A. Effect of propolis gel on the in vitro reduction of dentin permeability. J Appl Oral Sci. 2011; 19: 318- 23.

16. Markowitz K and Pashley DH. Discovering new treatments for sensitive teeth: the long path from biology to therapy. J Oral Rehab. 2008; 35: 300-15.

17. Assis JS and Rodrigues LK. Dentin Hypersensitivity after Treatment with Desensitizing Agents: A Randomized, Double-Blind, Split-Mouth Clinical Trial. Braz Dent J. 2011; 22: 157-61.

18. Addy M, West NX, Barlow A, Smith S. Dentin hypersensitivity: is there both stimulus and placebo responses in clinical trials? Int J Dent Hyg. 2007; 5:53-9.
19. Trowbridge HO and Silver DR. A review of current approaches to in-office management of tooth hypersensitivity. Dent Clin North Am. 1990; 34: 561- 81.

20. Ad Hoc Advisory Committee on Dentinal Hypersensitivity Council on Dental Therapeutics. Recommendations for evaluating agents for the reduction of dentinal hypersensitivity. J Am Dent Assoc 1986; 112: 709-10.

21. Pashley DH. Mechanism of dentin sensitivity. Dent Clin North Am. 1990; 34(3):449-73.

22. Krauser JT. Hypersensitive teeth, part II: treatment. J Prosth Dent. 1986; 56:307-11.

23. Narmatha V and Thakur S. An In-Vivo Comparative Study of the Efficacy of propolis, nano-Hydroxyapatite and potassium nitrate containing desensitizing agents. J Dent Sci. 2014; 2:113-18.

24. Clark GE and Troullos ES. Designing hypersensitivity clinical studies. Dent Clin North Am 1990; 34: 531-44. 